\title{
Three-Dimensional Spherical Shell Convection at Infinite Prandtl Number Using the 'Cubed Sphere' Method
}

\author{
J.W. Hernlund ${ }^{\mathrm{a}}$, P.J. Tackley ${ }^{\mathrm{a}, \mathrm{b}}$ \\ ${ }^{a}$ University of California, Department of Earth and Space Sciences, Los Angeles, \\ CA90095, USA \\ ${ }^{\mathrm{b}}$ University of California, Institute of Geophysics and Planetary Physics, Los Angeles, \\ CA90095, USA
}

Submitted to: Second M.I.T. Conference on Computational Fluid and Solid Mechanics, Sept. 30, 2002.

\begin{abstract}
We present a new finite difference code for modeling three-dimensional thermal convection in a spherical shell using the "cubed sphere" method of Ronchi et al. [1]. The equation of motion is solved using a poloidal potential formulation of the equation of motion for an iso-viscous, infinite Prandtl number fluid on a finite difference grid and advective transport is implemented using the $2^{\text {nd }}$-order MPDATA scheme of Smolarkiewicz [2]. Due to the high efficiency of multigrid acceleration, low memory requirements, and second-order accuracy of this model, we conclude that the cubed sphere method offers a great deal of potential for simulating complicated problems of fluid mechanics in spherical geometry.
\end{abstract}

\section{Introduction}

Highly viscous thermal convection in the rocky mantles of the solid planets is the primary process governing their thermal and mechanical evolution over long time scales. This process is driven primarily by the transfer of heat from the interior to the surface. Because thermal convection constitutes a non-linear problem, the main tool for studying finite amplitude convective motions is computer models. Due to the relative simplicity of the relevant equations in a parallel coordinate system, Cartesian domains are often used to obtain an approximation to convective motions in planetary mantles. This approximation has proven to be useful for many different problems, although it imposes a geometric symmetry between the upper and lower boundaries which does not exist in spherical geometry.

In the first spherical shell models the most popular method for solving the relevant equations were spectral, i.e., using spherical harmonics as basis functions. This allows a great deal of simplification of the equations (Chandrasekhar [3]), however, computation of the Legendre transforms can be expensive. The method's primary limitation, however, is that the elegance and simplicity are destroyed when more complicated effects, such as laterally-varying viscosity, are included, and viscosity varies by many orders of magnitude in planetary bodies. In addition, spectral methods do not offer a straightforward implementation on parallel computers since the basis functions are not local.

Recently, there has been an increase in the application of grid-based methods to mantle convection in spherical geometry. Methods such as finite elements and finite differences are local and can therefore more easily accommodate complex effects such as 
variable viscosity. They may also be decomposed for straightforward implementation on parallel computers, and thus offer the possibility of very high-resolution. Another benefit of grid-based methods is that in most cases it is possible to apply multigrid acceleration to the solution of the resulting large sets of equations.

Baumgardner [4] developed a finite element spherical shell convection code using a progressively refined icosahedral projection onto the sphere. In the 1990s this code was expanded to include the effects of variable viscosity (Yang [5]). Ratcliff et al. [6] developed a finite difference model of thermal convection on a spherical polar grid that included the effects of temperature dependent viscosity. This code was capable of handling high viscosity contrasts in a spherical shell, however its usefulness was limited by the coordinate singularity at the poles.

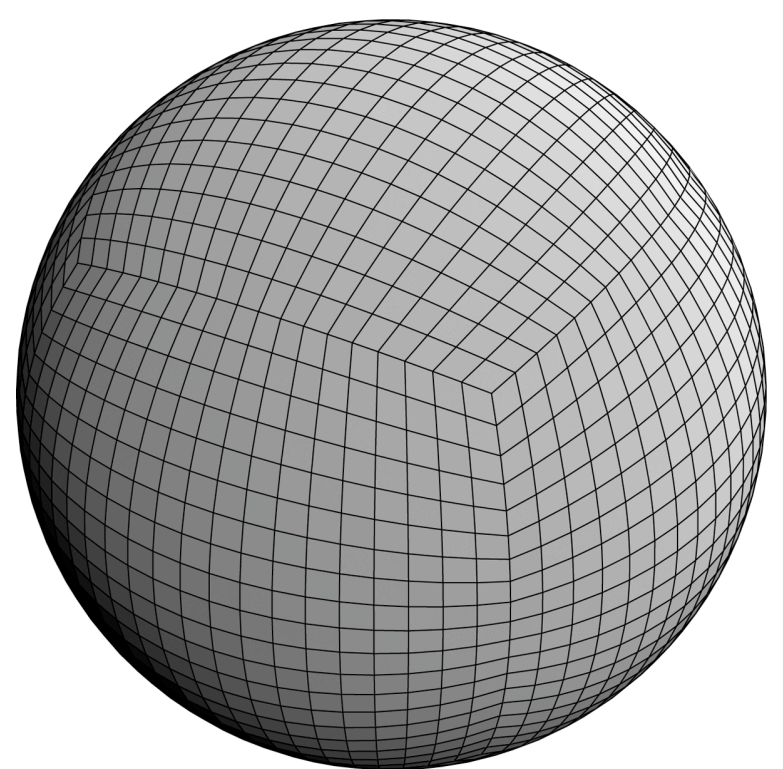

Figure 1: The horizontal coordinate lines of the cubed sphere grid on a spherical surface.

Ronchi et al. [1] proposed a new method for the solution of partial differential equations in spherical geometry consisting of projecting a cube onto a sphere to produce six separate patches that are then subdivided. The coordinate lines on each patch correspond to great circles that are perpendicular at the centers of each block (see figure 1). For a finite difference grid constructed on these patches, a suitable choice for coordinates insures that horizontal ghost points for every block lie in the same plane as the interior grid points of neighboring blocks. If the same radial discretization is maintained on each block, then ghost point exhanges can be made using simple onedimensional interpolations. The advantages of this technique are that each grid block contains no singularities, exhibits a nearly constant horizontal spatial coverage, and coupling the blocks requires a negligible fraction of the total computational effort.

\section{Basic Equations}

The equation of motion for an incompressible, infinite Prandtl number, isoviscous fluid in the Boussinesq approximation is, 


$$
\vec{\square} \square \vec{\square} \square \vec{\square} \square \vec{v}=R a \vec{\square} \square(T \vec{\square}),
$$

where $\vec{\Pi}$ is the gradient vector, $\vec{v}$ is the velocity vector, $T$ is the temperature, $\vec{\Pi}$ is the unit "up" vector, and $R a$ is the thermal Rayleigh number. It has been shown (Chandrasekhar [3]) that for this style of convection the solenoidal velocity field can be written exclusively in terms of a poloidal potential $W$,

$$
\vec{v}=\vec{\square} \square \vec{\square} \square(W \vec{D}) .
$$

Finally, the conservation of energy requires that,

$$
\frac{\partial T}{\partial t}+\square \cdot(\vec{v} T)=\square^{2} T .
$$

Explicit expressions for the curl, gradient, and divergence using the physical components of a vector are given in Ronchi et al. [1].

\section{Numerical Technique}

Eq (1) is solved in terms of a poloidal potential using two second-order equations that satisfy homogeneous boundary conditions on the inner and outer boundaries. This approach simplifies the treatment of the boundary conditions since it only requires one level of ghost points at the sides of each coordinate block. The finite difference equations are relaxed using multigrid V-cycles with a Gauss-Seidel red-black smoother at each grid level. When the number of radial points is chosen to be equal to half the number of horizontal grid points on each block, the residual root-mean-square error decreases by a factor of 5 to 9 for each V-cycle depending on the initial guess. This is equivalent to the residual reduction observed for a Poisson solver in Cartesian coordinates using the same method.

Time-integration is treated separately from the equation of motion using $\mathrm{Eq}$ (3). An accurate treatment of advective transport is a challenging problem in numerical modelling. In addition to the usual concerns of implementing advection on an Eulerian grid, the cubed sphere coordinate system is both curvilinear and non-orthogonal. As a result, the application of ordinary finite difference schemes contains subtle complications. Upwind advection schemes offer stability as well as global conservation of the advected field, and therefore have desirable qualities that are relevant to the problem of convective heat transfer. The main source of error in upwind schemes is related to "numerical dissipation," which is caused by truncation of higher order terms in the finite difference equations. In non-orthogonal, curvilinear coordinates, this numerical dissipation is anisotropic and spatially variable, which leads not only to dissipation of the advected field but also creates significant grid-related distortion. A truncation analysis of a simple first-order upwind scheme in a non-orthogonal grid reveals that this numerical diffusivity is greatest along coordinate lines. We correct for the second order spatial and temporal truncation errors using the MPDATA technique of Smolarkiewicz [2], and find that it effectively removes the undesired effects of the upwind advection technique on the cubed sphere grid.

\section{Results and conclusions}

Planforms for steady cubic and tetrahedral cases and a time-dependent case are shown in figure 2 . The time-dependent case uses only a single coordinate block with periodic boundary conditions. 


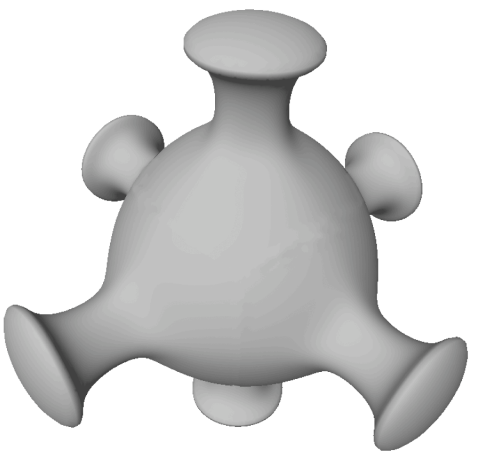

(a)

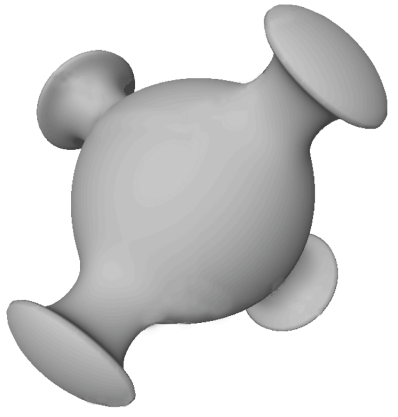

(b)

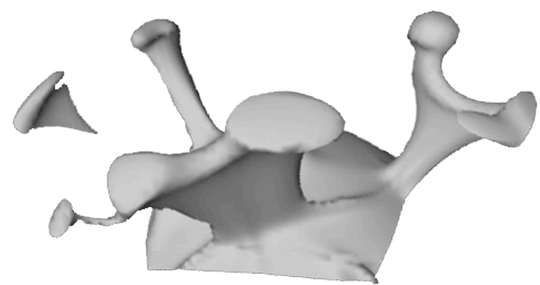

(c)

Figure 2: Isosurfaces of constant temperature $(T=0.5)$ for the steady (a) tetrahedral and (b) cubic planforms at $\mathrm{Ra}=7000$ and (c) time-dependent convection in a single block at $\mathrm{Ra}=500000$.

Figure 3 shows the scaling of two global characteristics with Rayleigh number. The Nusselt number is defined as the heat flow relative to the conductive heat flow (in the absence of convective motions).
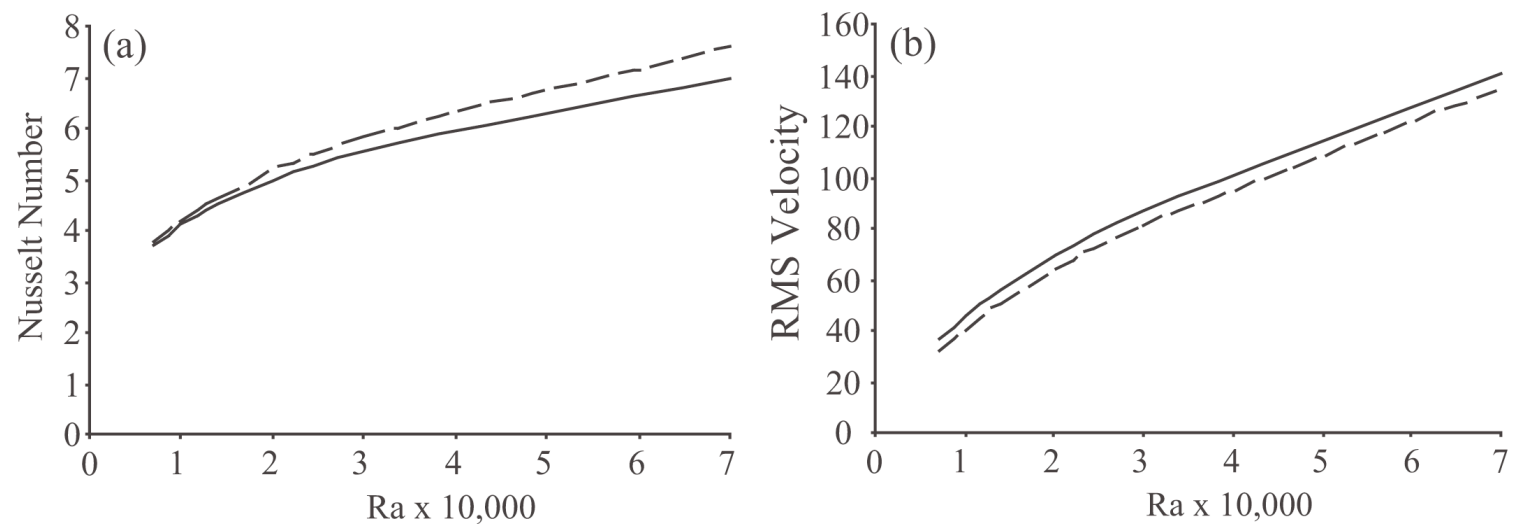

Figure 3: (a) Nusselt number and (b) root-mean-square velocity vs. Rayleigh number for the cubic (dashed line) and tetrahedral (solid line) planforms of steady-state convection in a spherical shell with a radius ratio of 0.55 .

The presented values are within a few percent of the those obtained by Bercovici et al. [7], and follow the same pattern and relative relationships. Test runs with both patterns exhibit a slight increase in Nusselt number as the resolution is increased. At low resolutions, the Nusselt numbers obtained are almost indistinguishable from the results of Bercovici et al. [7]. The results shown in figure 3 are given for a calculation having a vertical resolution of 32 radial grid points. 


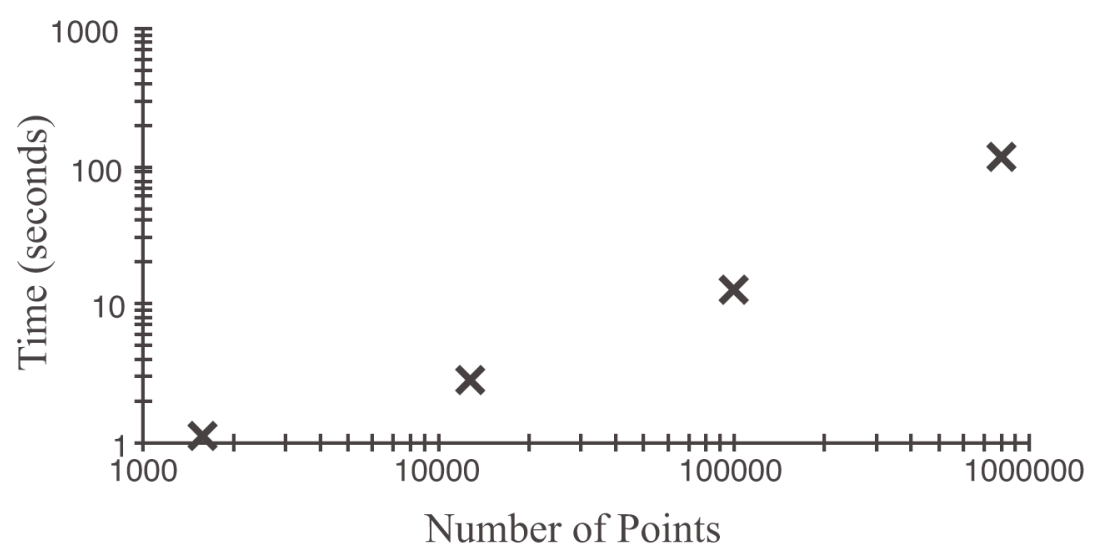

Figure 4: Run time in seconds for the first 10 timesteps in a full sphere as a function of the number of grid points on a single Athlon XP $1.9 \mathrm{GHz}$ processor.

Figure 4 shows the CPU time on a single Athlon XP $1.9 \mathrm{GHz}$ processor required for the first 10 timesteps as a function of the number of grid points, and demonstrates the efficiency and scalability of this technique and its potential for handling high resolution grids.

\section{References}

[1] Ronchi C, Iacono R, Paolucci PS. The "cubed sphere": a new method for the solution of partial differential equations in spherical geometry. Journal of Computational Physics 1996; 124, 93-114.

[2] Smolarkiewicz PK. A simple positive definite advection scheme with small implicit diffusion. Monthly Weather Review 1983; 111, 479.

[3] Chandrasekhar S. Hydrodynamic and hydromagnetic stability. Oxford University Press, 1961.

[4] Baumgardner JR. A three-dimensional finite element model for mantle convection. Ph.D. thesis, UCLA, 1983.

[5] Yang W. Variable viscosity thermal convection at infinite prandtl number in a thick spherical shell. Ph.D. thesis, University of Illinois, 1997.

[6] Ratcliff JT, Schubert G, Zebib A. Effects of temperature-dependent viscosity on thermal convection in a spherical shell. Physica D 1996; 97, 242-252.

[7] Bercovici D, Schubert G, Glatzmaier GA, Zebib A. Three-dimensional thermal convection in a spherical shell. Journal of Fluid Mechanics 1989; 206, 75-104. 